\title{
El Departamento de Ventas como generador de ingresos en las Pymes de Guayaquil, Ecuador.
}

\section{The Sales Department as an income generator in the SMES of Guayaquil, Ecuador.}

\author{
Lic. Ángel Fortunato Bernal Álava 1*, Abg. Paco Egdon Granoble Chancay 22, \\ 1.* Doctor en Educación, Universidad Estatal del Sur de Manabí, Jipijapa, Ecuador. Email: angel.bernal@unesum.edu.ec \\ ORCID: http://orcid.org/0000-0002-9212-1234
}

2. Magister en Contabilidad y Auditoria, Universidad Estatal del Sur de Manabí, Jipijapa, Ecuador. Email: paco.granoble@unesum.edu.ec ORCID: https://orcid.org/0000-0002-3243-5418

Destinatario: angel.bernal@unesum.edu.ec

Recibido: 18/ enero/2021 Aceptado: 17/febrero/2021 Publicado: 31/marzo/2021

Como citar: Bernal Álava, Ángel F., \& Granoble Chancay, P. E. (2021). El Departamento de Ventas como generador de ingresos en las Pymes de Guayaquil, Ecuador. Revista E-IDEA Journal of Business Sciences, 3(9), 26-35. https://doi.org/10.53734/eidea.vol3.id85

Resumen: Las pequeñas y medianas empresas del Ecuador (Pymes) dedicadas al comercio en diferentes sectores económicos constituyen una fuente de empleo y productividad para el país. Estas empresas son en su mayoría manejadas de manera informal con diversas fallas en los procesos internoscomo consecuencia de la falta deplanificación, organización y estructuración del Departamento de Ventas que dificultan su rentabilidad y consideración para adquirir fuentes de financiamiento que mejoren la producción y aumento de las ventas. De acuerdo a este contexto, se llevó a cabo una investigación descriptiva con una revisión documental sobre los aspectos básicos y necesarios de la estructura y organización del Departamento de Ventas como generador de ingresos en las Pymes de Guayaquil, Ecuador. Entre los hallazgos se infiere que las reconocidas contribuciones de las Pymes en la economíaecuatoriana no son suficientes para su fácil acceso a fuentes de financiamiento por no cumplir con los estándares de garantías y requerimientos que solicitan la mayoría de las instituciones financieras, quienes limitan sus créditos por su falta de rentabilidad. Por ello, la implementación de técnicas y estrategias de ventas que exalten las cualidades de los productos ofrecidos mejoran el pronóstico de ventas y aumentan ingresos, así como el desarrollo productivo y socioeconómico y rentabilidad de las Pymesen Guayaquil.

Palabras Clave: Departamento de Ventas, ingresos por ventas, Pymes.

Abstract: Ecuador's small and medium-sized enterprises (SMEs) engaged in commerce in different economic sectors constitute a source of employment and productivity for the country. These companies are mostly managed informally with various failures in the internal processes as a consequence of the lack of planning, organization and structuring of the Sales Department that hinder their profitability and consideration to acquire financing sources that improve production and increase of sales. According to this context, a descriptive investigation was carried out with a documentary review on the basic and necessary aspects of the structure and organization of the Sales Department as an income generator in SMEs in Guayaquil, Ecuador. Among the findings, it is inferred that the recognized contributions of SMEs in the Ecuadorian economy are not enough for their easy access to financing sources because they do not comply with the guarantee standards and requirements requested by most financial institutions, who limit their credits. for its lack of profitability. Therefore, the implementation of sales techniques and strategies that enhance the qualities of the products offered improve the sales forecast and increase income, as well as the productive and socioeconomic development and profitability of SMEs in Guayaquil.

Keywords: Sales Department, sales revenue, SMEs.

Bernal Álava, Ángel F., \& Granoble Chancay, P. E. (2021). El Departamento de Ventas como generador de ingresos en las Pymes de Guayaquil, Ecuador. E-IDEA Journal of Business Sciences, 3(9), 26-35. 


\section{INTRODUCCIÓN}

$\mathrm{E}$ n el Ecuador, las Pymes son pequeñas y medianas empresas por su número de empleados, valor bruto en ventas anuales y valor de los activos totales, dedicadas al comercio al por mayor y al por menor, con diferentes actividades económicas en diversos sectores económicos y constituyen una fuente de empleo y productividad para el país.

En su gran mayoría, las Pymes comienzan como empresas familiares manejadas de manera informal con diversas fallas en los procesos internos que evidencian la poca planificación financiera (Baquero Piloso y Parrales Cobos, 2018) que dificultan su rentabilidad y consideración para adquirir fuentes de financiamiento que mejoren la producción y aumento de las ventas.

En ese sentido, la falta de planificación, organización y estructuración del Departamento de Ventas de las Pymes de Guayaquil, la ciudad con mayor número de Pymes en el Ecuador ubicada en la provincia Guayas (Superintendencia de Compañías, Valores y Seguros, 2020), han traído como consecuencia la falta de liquidez e incapacidad de las Pymes de costear sus altos gastos operativos.

De allí, que en la presente investigación se describan los aspectos básicos y necesarios de la estructura y organización del Departamento de Ventas como generador de ingresos en las Pymes de Guayaquil frente a los problemas que se están suscitando desde el punto de vista financiero de las Pymes.

\section{METODOS}

En el presente estudio se analiza el rol del departamento de ventas como generador de ingresos en las Pymes de Guayaquil, Ecuador, mediante una investigación descriptiva bajo el enfoque cuantitativo con una revisión documental sobre estructura y funciones del departamento de ventas como generador de ingresos de las pequeñas y medianas empresas de Guayaquil, Ecuador.

\section{RESULTADOS Y DISCUSIÓN}

Las ventas anuales de una empresa permiten su clasificación como micro, pequeñas, medianas y grandes empresas. Las pequeñas y medianas empresas (Pymes) han tenido una creciente importancia en América Latina puesto que contribuyen a la creación de empleo, aumento de la productividad e ingresos, según Moreno Rodríguez, Cevallos Villegas y Balseca Villavicencio(2018), las Pymes se definen como "un conjunto de pequeñas y medianas empresas que de acuerdo a su volumen de ventas, capital social, cantidad de trabajadores, y su nivel de producción o activos presentan características propias de este tipo de entidades económicas" (p. 303). 


\section{E-IDEA}

J OURNAL OF B USINESS SCIENCES

Específicamente en el Ecuador, las Pymes se clasifican según el número de trabajadores, valor bruto en ventas anuales y el valor en activos totales (Tabla 1), son consideradas versátiles por su facilidad de adaptarse a los diferentes cambios de capital que se puedan presentar, asimismo "se les atribuye el mayor número de innovaciones tecnológicas y colocaciones de producción nacional en mercados extranjeros" (Avalos, 2020, p. 2). En las últimas cifras, se conoce que existen más de 6 mil Pymes activas en el Ecuador, las cuales representan el $14 \%$ de las empresas a nivel nacional que desempeñan actividades económicas relacionadas con el comercio, industrias manufactureras y agricultura que generan alrededor de USD 1 Millón a 5 Millones cada año (Moreno Rodríguez, Cevallos Villegas y Balseca Villavicencio, 2018).

Tabla 1

Calificación de las Pymes en el Ecuador:

\begin{tabular}{lllllr}
\hline Clasificación & Micro & Pequeñas & Medianas & \multicolumn{2}{l}{ Grandes } \\
\hline$N^{\circ}$ de empleados & 1 a 9 & 10 a 49 & 50 a 199 & Mayor que 200 \\
\hline Valor Bruto en & Pequeñas & De 100 Mil a & De 1 Millón & Más de & 5 \\
Ventas Anuales & & 1 Millón & a 5 Millones & Millones & \\
\hline Valor de los & Menos que & De 100 Mil a & De 750 Mil a & Más de 4 & \\
Activos Totales & 100 Mil & 750 Mil & 4 Millones & Millones & \\
\hline
\end{tabular}

Fuente: Superintendencia de Compañías, Valores y Seguros (2020)

Las Pymes se concentran en un 37,85\% en la provincia Guayas, 36,75\% en Pichincha, 5,14\% en Azuay, 4,60\% en El Oro, 3,96\% en Manabi y 11,70\% en otras provincias, de las cuales el 35,32\% se dedica al comercio al por mayor y al por menor, reparación de vehículos automotores y motocicletas; $12,5 \%$ a industrias manufactureras, $12,45 \%$ a la agricultura, ganadería, silvicultura y pesca; 7,43\% a transporte y almacenamiento; $6,04 \%$ a actividades de servicios administrativos y de apoyo y 26,26\% a otras actividades (Aval, 2019). En la tabla 2 se presenta el número de empresas según tamaño de la empresa, ventas totales según participación que registraron en su declaración al SRI, plazas de empleo registraron en el IEES y masa salarial o suma total de remuneraciones registradas en las empresas, en el Ecuador

\section{Tabla 2}

Estadísticas de Pymes en el Ecuador:

\begin{tabular}{|c|c|c|c|c|}
\hline Clasificación & Micro & Pequeñas & Medianas & Grandes \\
\hline $\begin{array}{c}\text { No de } \\
\text { empresas }\end{array}$ & 816.553 & 64.117 & 8.529 & 4.260 \\
\hline $\begin{array}{c}\text { Ventas totales } \\
\text { Plaza de } \\
\text { empleo }\end{array}$ & $\mathbf{\$ 1 . 5 7 4 . 3 7 9 . 2 1 9}$ & $\$ 26.715 .942 .725$ & $\$ 19.091 .411 .065$ & $\$ 121.675 .426 .932$ \\
\hline Masa salarial & $\$ 3.385 .460 .151$ & $\$ 3.855 .180 .184$ & $\$ 4.479 .123 .507$ & $\$ 14.265 .408 .745$ \\
\hline
\end{tabular}

Fuente: Superintendencia de Compañías, Valores y Seguros (2020) 
Las fuentes de financiamiento de las Pymes en el Ecuador son generalmente por créditos en el sistema bancario o a través de la utilización de capital propio y otra reducida porción de financiamiento proviene de aportes societarios, créditos del exterior o reinversión de utilidades (capital orgánico), estos créditos van desde \$50.000 hasta \$25 millones de dólares y las principales instituciones que se han dedicado a aportar grandes volúmenes de crédito a las Pymes son el Banco Central del Ecuador, el Banco del Estado y la Corporación Financiera Nacional (Avalos, 2020).

El logro de los créditos obligan a la producción y aumento de las ventas, de allí la importancia del departamento de ventas para generar ingresos en las Pymes, las cuáles poseen en su mayoría una deficiente o nula cultura financiera, debido a que, al menos en Guayaquil, la mayoría son empresas familiares y manejadas de manera informal y cuyos procesos internos poseen diversas fallas que se reflejan en la falta de liquidez y altos gastos operativos, como consecuencia de la poca planificación financiera caen y mal manejo de recursos, que no pueden ser cubiertos con las ventas (Baquero Pilosoy Parrales Cobos, 2018).

En ese sentido, es imperativo el aumento de ingresos de las Pymes de Guayaquil, ubicada en la provincia Guayas, donde se ubican la mayor cantidad de Pymes, mediante el aumento de ventas que permitan aumentar la liquidez mediante un Departamento de Ventas correctamente estructurado y organizado de acuerdo a las necesidades de las Pymes y las actividades económicas que desarrollan.

Para tal propósito, es necesariamente indispensable conocer la definición de ventas según el Comité de Definiciones de la Asociación Americana de Mercadeo (AMA, 2018), el cual define las ventas como la cantidad de dinero que gana una empresa durante un periodo de tiempo, como un año o trimestre fiscal. El concepto de ventas está estrechamente relacionado con el volumen de ventas, que se refiere al número de unidades que vende una empresa en un periodo determinado, debido a que las ventas totales equivalen al volumen de ventas multiplicado por el precio unitario. La estructura de ventas de una empresa básicamente se clasifica de la siguiente manera: 


\section{E-IDEA}

\section{Figura 1}

Ventas:

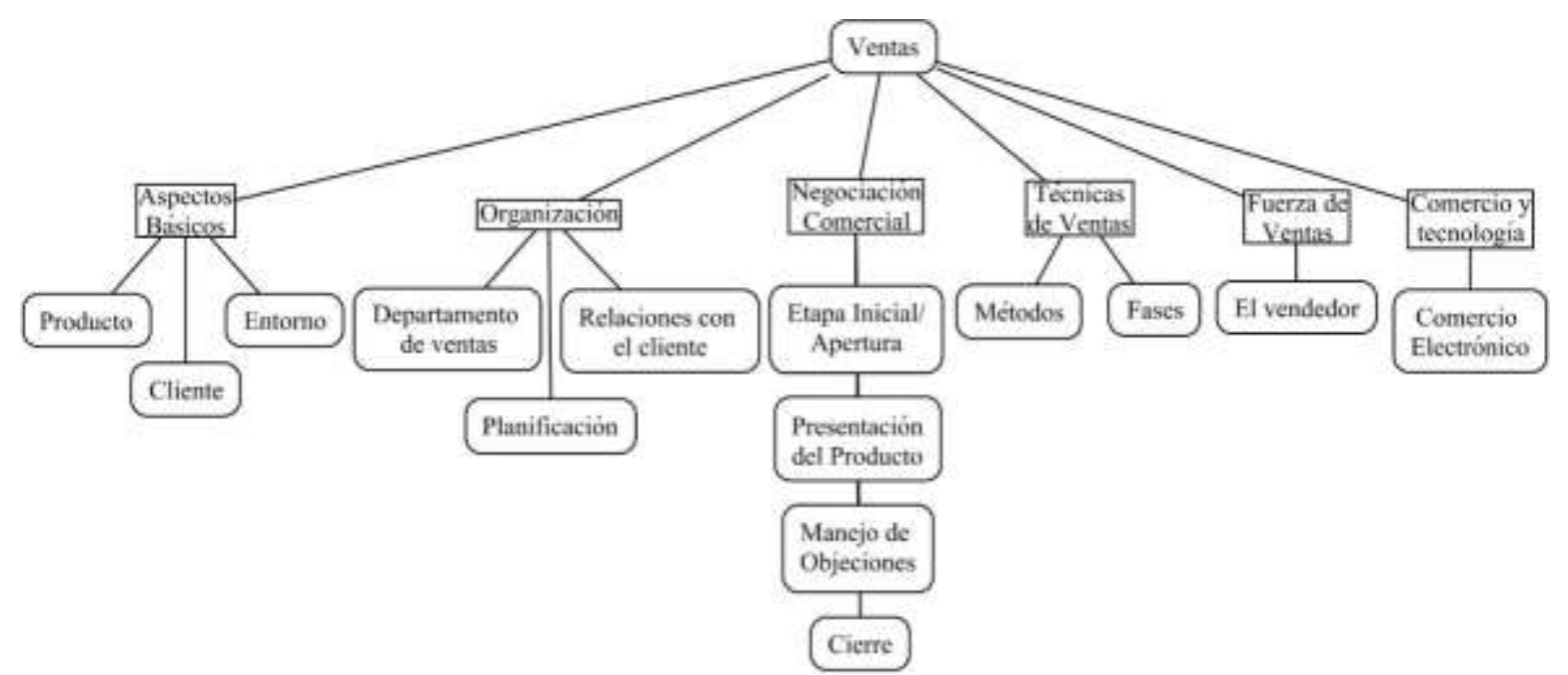

Fuente: Elaborado con datos de Navarro Mejía (2012)

Dentro de los aspectos básicos de las ventas, el producto (bien material, servicio o idea que satisface la necesidad del consumidor), de consumo, industrial o servicio, posee una serie de atributos (entre ellos su marca, calidad, diseño y diferenciación respecto a otros productos) necesarios para el cliente o consumidor, en un entorno externo (donde intervienen factores económicos, técnicos, políticos, socioculturales y ambientales) e interno (los cuales dependen de la empresa, como las metas, objetivos, su personal, recursos, capacidad de producción, capacidad de servicio y capacidades tecnológicas, de investigación y desarrollo) Navarro Mejía (2012).

Estos aspectos obligan a la organización del departamento de ventas, el cual se encarga de la planificación, dirección y control de las actividades del personal de ventas de una empresa, incluido el reclutamiento, la selección, la capacitación, el equipamiento, la asignación, el enrutamiento, la supervisión, el pago y la motivación de los empleados. La gestión de ventas implica tres procesos interrelacionados: formulación de un programa estratégico de ventas, implementación del programa de ventas; y evaluación y control del desempeño del departamento de ventas (AMA, 2018).

Los propósitos perseguidos por el departamento de ventas, dependen de su organización, estructura y su diferenciación respecto al departamento de marketing. Si bien la estructura del departamento de ventas puede ser horizontal, vertical, matricial y multidimensional, es la empresa la que define su estructura en función de los métodos que debe aplicar para satisfacer las necesidades de la organización, considerando como alternativas la geográfica (donde se divide geográficamente las ventas); por tipo de producto (cuando el departamento de ventas se estructura según las diferentes líneas de productos que produce); por cliente (mayorista, minorista, entre otros); y por función de 
ventas (cuando el vendedor fortalece distintas capacidades y habilidades en funciones de ventas) (Acosta Véliz, et al., 2018).

En ese sentido, los tipos de ventas directas o indirectas a través de intermediarios para distribuidores mayoristas, minoristas o agentes comerciales, comerciales y profesionales; y free line (particulares independientes), orientan la elección de las estrategias de ventas (bajar los precios, adicionar a las ventas premios o regalos, ofertas, crear servicios que vinculen al cliente durante un periodo de tiempo, buscar al cliente, estrategias de vencer el miedo en el ejercicio de la venta, estrategias del coaching de ventas, estrategias de las normas fijas y normas variables, estrategias de cambio cuando el producto o servicio no da resultado, estrategias de conocer bien a los clientes, estrategias del uso de redes sociales y estrategias del uso de testimonios que apoyan la venta) (Ramos Aranda, 2017); y las técnicas de ventas (Tabla 3) (Acosta Véliz, et al., 2018) donde se definen metas y objetivos, aplican políticas, establecen procedimientos e ideas estratégicas, dirigen tácticas y diseñan controles según cada técnica mediante los resultados de un estudio previo sobre las oportunidades del mercado con la finalidad de obtener ganancias para la empresa en un tiempo determinado.

\section{Tabla 2}

Estadísticas de Pymes en el Ecuador: TÉCNICA DE VENTA

\section{DEFINICIÓN}

Hace referencia a las siglas Atención, Interés, Deseo y Acción, promoviendo en el

Modelo AIDA potencial cliente estas manifestaciones positivas hacia el producto o servicio. Se recomienda para ventas sencillas.

Se enfoca en generar preguntas para lograr una secuencia que promueve el interés

Técnica SPIN hacia la compra. Se recomienda para ventas complejas. Sus pasos son: analizar la situación, detectar los problemas, conocer la importancia del problema, presentar una necesidad de solución, promover el interés por la propuesta.

Consiste en la atención, interés, convicción, deseo y cierre, donde se incorporan a

Técnica AICDC la técnica AIDA, la convicción, donde el vendedor tiene el reto de convencer al cliente que comprar el producto será la mejor decisión.

Presenta un análisis de la palabra ventas a través de los siguientes pasos: verificar

SistemaZelev Noel preparativos, entrevista efectiva y vendedora, necesidades establecidas previamente, tarea de demostración y satisfacción total y atención postventa.

Se refiere a promocionar los productos a un grupo de interés de tal manera que no

Técnica Promoción se pierda tiempo promocionando en otros nichos de mercado que son la población que atiende el producto actual.

Venta enlatada

El vendedor usa la misma estrategia para cualquier venta, el éxito de ella es la naturalidad de su actuación en cualquiera de los casos.

El vendedor enfatiza sus esfuerzos en conocer las necesidades del cliente y luego

Venta consultiva ofrecer el producto que se adapte a sus intereses. Es común en operaciones de tipo industrial o de alta especialidad.

Consiste en la reposición, reparación y/o reembolso que el vendedor puede

Técnica de las tres Rs ofrecer al cliente prospecto, convirtiéndose las tres Rs en un poderoso argumento de ventas.

Venta adaptativa $\quad$ Esta técnica se adapta al vendedor y al cliente, no se usa la misma técnica, el cliente participa de forma muy activa.

Fuente: Elaborado con datos de Acosta Véliz, et al., (2018) y Ramos Aranda (2017) 
Aunque muchas de estas técnicas de ventas tienen décadas desde que fueron creadas aún continúan siendo aplicadas y actualmente sustentan técnicas de ventas que emplean tecnologías Smart, como la Inteligencia Artificial, cloudcomputing, Internet ofThings y Big data, que facilitan el proceso de registro de la información sobre clientes, vendedores, despachadores, producción y administradores del negocio con la finalidad de mejorar la productividad a la empresa (Barrientos-Avendaño, et al, 2019), por tal razón los sistemas de información digital ayudarán a las empresas a "mantenerse a futuro y lograr una ventaja competitiva dentro de una economía mundial globalizada" (Proaño, Orellana y Martillo, 2018, p. 23).

El proceso de la venta comienza con el primer contacto con el cliente, previo a la presentación o demostración del producto en el que se resalten los atributos, ventajas y beneficios que permitirán reducir las objeciones del cliente a realizar la compra y concluir de manera satisfactoria la negociación, dividido por fases: preventa (momentos antes de proceder con los pasos de la venta); venta (negociación con el cliente, demostración); y postventa (soporte técnico, garantías, capacitaciones, atención al cliente) (Ramos Aranda, 2017).

Cabe resaltar el rol protagónico del personal del departamento de ventas en el éxito de las ventas de una empresa, caracterizados por su autonomía, creatividad, emprendimiento, y otro conjunto de habilidades requeridas para cumplir con todas las exigencias del departamento, adicionalmente, el gerente de ventas debe poseer liderazgo y la capacidad de motivar y comprometer a su equipo en el logro de los objetivos planteados (Acosta Véliz, et al., 2018), especialmente cuando sus funciones adquieren mayor responsabilidad mientras la empresa va creciendo y aumentando sus ventas.

De lo anteriormente expuesto, se infiere que la organización, estructuración y planificación del Departamento de Ventas permite "eliminar la informalidad, incrementar los volúmenes de producción, elevar la asociatividad y procurar un mayor acceso al crédito productivo" (Cortés Solís, 2020, p. 23), por lo que el departamento de ventas debe sintetizar y sistematizar sus funciones en operaciones prácticas y contundentes respecto a las metas y objetivos planteados para analizar las oportunidades del mercado mediante la revisión de las características del mercado, la competencia, ventas, costos, beneficios, sistemas de distribución(Acosta Véliz, et al., 2018) que servirán para elaborar mejores pronósticos de ventas con los cuales se seleccionarán las mejores estrategias para el negocio para continuar hacia el desarrollo y seguimiento de las actividades de ventas e ingresos.

De esta manera se puede aumentar la rentabilidad de las Pymes en Guayaquil, es decir, que los ingresos recibidos por las ventas sean mayores que sus gastos, por lo que el éxito de las Pymes consiste en mantener los costos bajo control y garantizar que los ingresos sean suficientes para financiar el crecimiento mediante la planificación de estrategias de ventas y un adecuado manejo de los clientes (OncebaySacsara, 2019).Asimismo, el gerente de ventas debe desarrollar continuamente políticas de venta que capten nuevos clientes y

Bernal Álava, Ángel F., \& Granoble Chancay, P. E. (2021). El Departamento de Ventas como generador de ingresos en las Pymes de Guayaquil, Ecuador. E-IDEA Journal of Business Sciences, 3(9), 26-35. 
amplíen la plaza comercial con la finalidad de generar mayor producción y obtener mayores ingresos para la empresa..

\section{CONLUSIONES}

A pesar de las reconocidas contribuciones de las Pymes en la creación de empleos, aumento de la productividad e ingresos del Ecuador, así como su facilidad de adaptarse a los diferentes cambios de capital, innovaciones tecnológicas y colocaciones de producción nacional en mercados extranjeros, las Pymes tienen grandes dificultades para acceder a fuentes de financiamiento debido a que no pueden cumplir con los estándares de garantías y requerimientos que solicitan la mayoría de las instituciones financieras. Estos requerimientos tienen que ver con garantías de tipo hipotecario (terrenos, propiedades) o prendario (maquinarias, equipos) con porcentaje de cobertura requerido es de al menos 125\% y tasa de intereses de $9.75 \%$ la cual es reajustable en base a la Tasa Pasiva Referencial del Banco Central del Ecuador (Avalos, 2020)

Por otro lado, la falta de planificación, organización y estructuración del Departamento de Ventas de las Pymes en Guayaquil, por tratarse en su gran mayoría de empresas familiares que no invierten en la estructuración y planificación financiera, generanbajas ventas que no pueden cubrir su liquidez y gastos operativos, debido a que no aprovechan adecuadamente los proyectos de financiamiento bancarios y del Gobierno ecuatoriano.

Por ello, la implementación de técnicas y estrategias de ventas que exalten las cualidades de los productos ofrecidos por la empresa podrá mejorar el pronóstico de ventas al ser realizado con base a movimientos reales de las ventas de los clientes lo que permitirán mejoras en los procesos de planificación de demanda, compras, producción y distribución. Estas técnicas deben ir acompañadas de modelos de gestión, innovación y uso de tecnologías para para garantizar una mejor gestión, control, y toma de decisiones que puedan elevar el desarrollo productivo y socioeconómico de las Pymes.

La organización, estructuración y planificación del Departamento de Ventas son indispensables para la selección de las mejores técnicas y estrategias de ventas con las que se conseguirán ventas e ingresos que determinen la rentabilidad de las Pymes, por lo que captar nuevos clientes y ampliar la plaza comercial con la finalidad de generar mayor producción y obtener mayores ingresos deben ser los principales objetivos del Departamento de Ventas de las Pymes en Guayaquil. 


\section{E-IDEA}

J OURNAL OF B USINESS SCIENCES

\section{REFERENCIAS BIBLIOGRÁFICAS}

Acosta Véliz, M., Salas Narváez, L., Jiménez Carcado, M., y Guerra Tejada, A. (2018). La administración de ventas. Conceptos clave en el siglo XXI. Recuperado de https://www.3ciencias.com/wp-content/uploads/2018/02/La-administracion-deventas.pdf

American Marketing Association (AMA) (2018). Definition of Sales. Recuperado de: https://marketing-dictionary.org/s/sales/

Aval (2019). ¿Cuál es la situación financiera de las Pymes en el Ecuador? Recuperado de https://www.aval.ec/informacion-general-de-empresas/cual-es-la-situacionfinanciera- de-las-pymes-en-el-ecuador/

Avalos, G. (2020). Pymes en el Ecuador. Recuperado de https://plusvalores.com.ec/wpcontent/uploads/2020/06/Analisis-sobre-Pymes-en-Ecuador..pdf

Baquero Piloso, L., y Parrales Cobos, M. (2018). Evaluación financiera para PYMES exportadoras de productos no tradicionales de Guayaquil (tesis de pregrado).

Universidad de Guayaquil, Ecuador. Recuperado dehttp://repositorio.ug.edu.ec/handle/redug/37295

Barrientos-Avendaño, E., Coronel-Rojas, L., Cuesta-Quintero, F., y Rico-Bautista, D. (2019). Sistema de administración de ventas tienda a tienda: aplicando técnicas de inteligencia artificial.Revista Ibérica de Sistemas e Tecnologias de Informação 27, 677-689. Recuperado de: https://www.researchgate.net/

Cortés Solís, J. (2020). Modelo de optimización para la entrega de créditos destinados a fomentar el emprendimiento de Pymes de manufactura diversa, caso de estudio 
Guayaquil. (tesis de postgrado). Universidad tecnológica Empresarial de Guayaquil, Ecuador. Recuperado de http://181.39.139.68:8080/handle/123456789/1151

Moreno Rodríguez, C., Cevallos Villegas, D., y Balseca Villavicencio, I. (2018). Diseño de un modelo de cadena de valor para las Pymes en la ciudad de Guayaquil. Universidad y Sociedad, 10(5), 301-312. Recuperado de http://rus.ucf.edu.cu/index.php/rus

Navarro Mejía, M. (2012). Técnicas de Ventas. Recuperado de http://www.aliat.org.mx/BibliotecasDigitales/economico_administrativo/Tecnicas_ de_venta.pdf

OncebaySacsara, K. (2019).Financiamiento, Rentabilidad y Tributación de la micro y pequeña empresa del sector comercio, Rubro Plastiquería "Inversiones Ikiu" S.R.L(tesis de pregrado). Universidad Católica Los Ángeles Chimbote, Ayacucho, Perú. Recuperado de http://repositorio.uladech.edu.pe/handle/123456789/14830

Proaño, M., Orellana, S., y Martillo, I. (2018). Los sistemas de información y su importancia en la transformación digital de la empresa actual. Revista Espacios, 39(45).

Recuperado de:

https://www.revistaespacios.com/a18v39n45/18394503.html

Ramos Aranda, S. (2017). Técnicas de Ventas.

Recuperado de https://digitk.areandina.edu.co/

Superintendencia de Compañías, Valores y Seguros (2020). Ranking empresarial. Recuperado de https://appscvs.supercias.gob.ec/rankingCias/ 